\title{
Diagnostic Accuracy of Interleukin-27 between Tuberculous Pleural Effusion and Malignant Pleural Effusion: A Meta-Analysis
}

\author{
Qing Liu Yan-Xia Yu Xiao-Juan Wang Zheng Wang Zhen Wang \\ Department of Respiratory and Critical Care Medicine, Beijing Institute of Respiratory Medicine and \\ Beijing Chaoyang Hospital, Capital Medical University, Beijing, China
}

\section{Keywords}

Interleukin-27 · Tuberculous/malignant pleural effusion ·

Meta-analysis

\begin{abstract}
Background: The concentration assay of pleural effusion interleukin-27 (IL-27) has raised concern for diagnosing tuberculous pleurisy. Compared with malignant pleural effusion (MPE), the concentration of IL-27 in tuberculous pleural effusion (TPE) increased significantly. Accurate differentiating diagnosis is essential for choosing treatment for pleural effusion. Objective: The present meta-analysis is aimed at determining the accuracy of IL-27 in the differential diagnosis between TPE and MPE. Material and Method: After having retrieved the published studies, we combined the sensibility (SEN), specificity (SPE), positive likelihood ratio (PLR), negative likelihood ratio (NLR), and diagnostic odds ratio (DOR) of IL-27 in the diagnosis of TPE compared to MPE using a fixedeffect model. The summary receiver operating characteristic curve was applied to estimate the overall test performance. Results: In total, 550 patients (285 patients with TPE and 265 patients with MPE), included in 7 case-control studies, were enrolled. The summary assessments for IL-27 in the diagnosis between TPE and MPE were: SEN 0.93 (95\% CI 0.90-0.96), SPE 0.97 (95\% CI 0.94-0.98), PLR 25.88 (95\% CI 13.84-48.39),
\end{abstract}

NLR 0.07 (95\% Cl 0.05-0.11), and DOR 333.26 (95\% CI 146.10760.19), respectively. The maximal joint SEN and SPE was 0.95; the area under the curve was 0.99. Conclusion: IL-27 determination is a relatively accurate test for the diagnosis of TPE, which has very high SEN and SPE for discriminating TPE from MPE. The results of IL-27 assays should be interpreted in parallel with clinical findings and the results of conventional tests.

(c) 2018 S. Karger AG, Basel

\section{Introduction}

In clinical practice, pleural effusion (PE) is a usual disease or complication with multiple recognized etiologies including cancer, pulmonary infection, organ dysfunction and systemic conditions [1]. According to Light's criteria, pleural fluid is always divided into transudative and exudative PE. It has been well documented that tuberculosis and cancer represent the two most frequent causes of exudative PE with predominantly lymphocytes in pleural fluid and similar results of biochemistry and routine examination. Given there is a big gap between

Qing Liu and Yan-Xia Yu contributed equally to the present work.

\section{KARGER}

(c) 2018 S. Karger AG, Basel

E-Mail karger@karger.com

www.karger.com/res
Zhen Wang, MD, PhD

Department of Respiratory and Critical Care Medicine Beijing Chaoyang Hospital, Capital Medical University 8 GongtiNanlu, Chaoyang District, Beijing 100020 (China) E-Mails wz_0316@126.com 


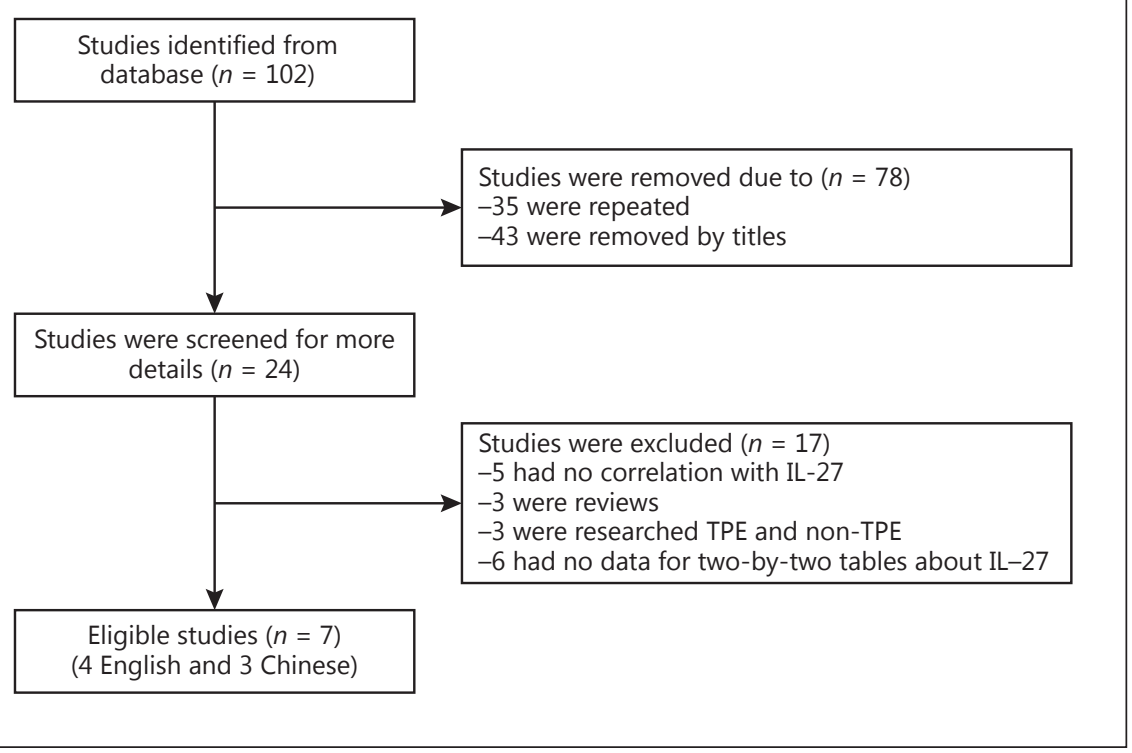

Fig. 1. Flow diagram of selected studies.

therapy and prognosis, identifying tuberculous pleural effusion (TPE) and malignant pleural effusion (MPE) becomes especially important. Differential diagnosis of TPE from PE with the other etiologies, especially MPE, is always a diagnostic challenge [2,3]. In a recent issue of Respiration, Yang et al. [4] put forward that the combinations of 2 or more tumor markers are highly specific but insufficiently sensitive for diagnosing MPE. The gold standard of MPE still relies on pathological diagnosis - to find malignant cells in $\mathrm{PE}$ or to find pathological changes on pleural biopsy specimen. The diagnosis of tuberculous pleurisy is given priority over the confirmation of Mycobacterium tuberculosis, combined with a pathological test, clinical feature, laboratory test, imaging presentation, and the responsiveness to antituberculosis chemotherapy. Medical thoracoscopy is the most precise procedure (specificity $100 \%$, sensitivity $98-100 \%$ ), with which we can directly find caseating granuloma, and it requires sufficient biopsy specimens; however, medical thoracoscopy is an invasive technique associated with various complications. Our previous publications investigated that adenosine deaminase (ADA) and interferon- $\gamma$, to a certain extent, both play roles in the diagnosis of TPE, but they failed to be regarded as reliable markers with satisfactory sensitivity and specificity. It is imperative to find out novel pleural markers to make a definite diagnosis of TPE and MPE.

Interleukin-27 (IL-27), as a new member of interleukin-12 (IL-12) families, has increased interest in its inves- tigation in recent years. It is identified as a heterodimeric cytokine composed of the Epstein-Barr virus-induced gene 3 and the p28 subunit [5]. IL-27 is mainly secreted by antigen-presenting cells [6] and regulates various immune diseases through its dual pro-inflammatory and anti-inflammatory effects on immune responses. IL-27 is involved in malignant diseases [7] and infectious diseases $[8,9]$, the latter including severe septicemia and tuberculosis. Our previous studies put forward that IL-27 is likely to participate in the formation of PE [10], and plays an important role in immune responses initiated by $M$. $t u$ berculosis, and can further be of diagnostic value. In this meta-analysis, the accuracy of IL-27 in the differential diagnosis between TPE and MPE is of particular interest.

\section{Material and Method}

Search Strategy and Study Selection

Two investigators performed searches of the Wanfang, Weipu, CNKI, Pubmed, and Embase database up to December 13, 2017, using the keywords "interleukin-27/IL-27," "tuberculous pleural effusion/fluid," and "malignant pleural effusion/fluid." All retrievals were from English and Chinese language publications concerning human studies.

Publications were included if: (1) subjects were in the TPE and MPE contrast group; (2) publications provided data for two-bytwo tables or other relevant data (sensibility [SEN], specificity [SPE]). Two reviewers in charge of retrieval estimated the eligibility of the studies; however, the final decision was made by a third investigator if there was any divergence. The process of searching and selecting the studies is shown in Figure 1. 
Table 1. Characteristics and quality of 7 studies included in the meta-analysis

\begin{tabular}{|c|c|c|c|c|c|c|c|c|c|c|c|c|c|}
\hline \multirow[t]{2}{*}{ First author } & \multirow[t]{2}{*}{ Setting } & \multirow[t]{2}{*}{ Language } & \multirow[t]{2}{*}{ Method } & \multirow[t]{2}{*}{ Samples } & \multirow[t]{2}{*}{$\begin{array}{l}\text { Cutoff, } \\
\text { ng/L }\end{array}$} & \multicolumn{2}{|c|}{$\begin{array}{l}\text { Number of } \\
\text { patients }\end{array}$} & \multicolumn{4}{|c|}{ Test results } & \multicolumn{2}{|c|}{ Quality scores } \\
\hline & & & & & & TPE & MPE & $\mathrm{TP}$ & $\mathrm{FP}$ & $\mathrm{FN}$ & $\mathrm{TN}$ & QUADAS & STARD \\
\hline Yang [10], 2012 & China & English & ELISA & $\mathrm{PE}$ & 1007 & 68 & 63 & 63 & 1 & 5 & 62 & 10 & 16 \\
\hline Liu [27], 2016 & China & English & ELISA & $\mathrm{PE}$ & 921.5 & 35 & 46 & 33 & 1 & 2 & 45 & 10 & 15 \\
\hline $\mathrm{Wu}[25], 2013$ & China & English & ELISA & $\mathrm{PE}$ & 900.8 & 40 & 26 & 38 & 1 & 2 & 25 & 10 & 17 \\
\hline Niu [30], 2012 & China & Chinese & ELISA & $\mathrm{PE}$ & 846 & 23 & 21 & 23 & 1 & 0 & 20 & 11 & 13 \\
\hline Tang [31], 2017 & China & Chinese & ELISA & $\mathrm{PE}$ & 36.91 & 45 & 45 & 39 & 3 & 6 & 42 & 10 & 14 \\
\hline
\end{tabular}

PE, pleural effusion; TPE, tuberculous pleural effusion; MPE, malignant pleural effusion; TP, true-positive result; FP, false-positive result; FN, false-negative result; TN, true-negative result; QUADAS, the quality assessment for studies of diagnostic accuracy (maximum score 14); STARD, standards for reporting the accuracy of a diagnostic test (maximum score 25).

Table 2. Diagnostic methods of TPE and MPE for each studies

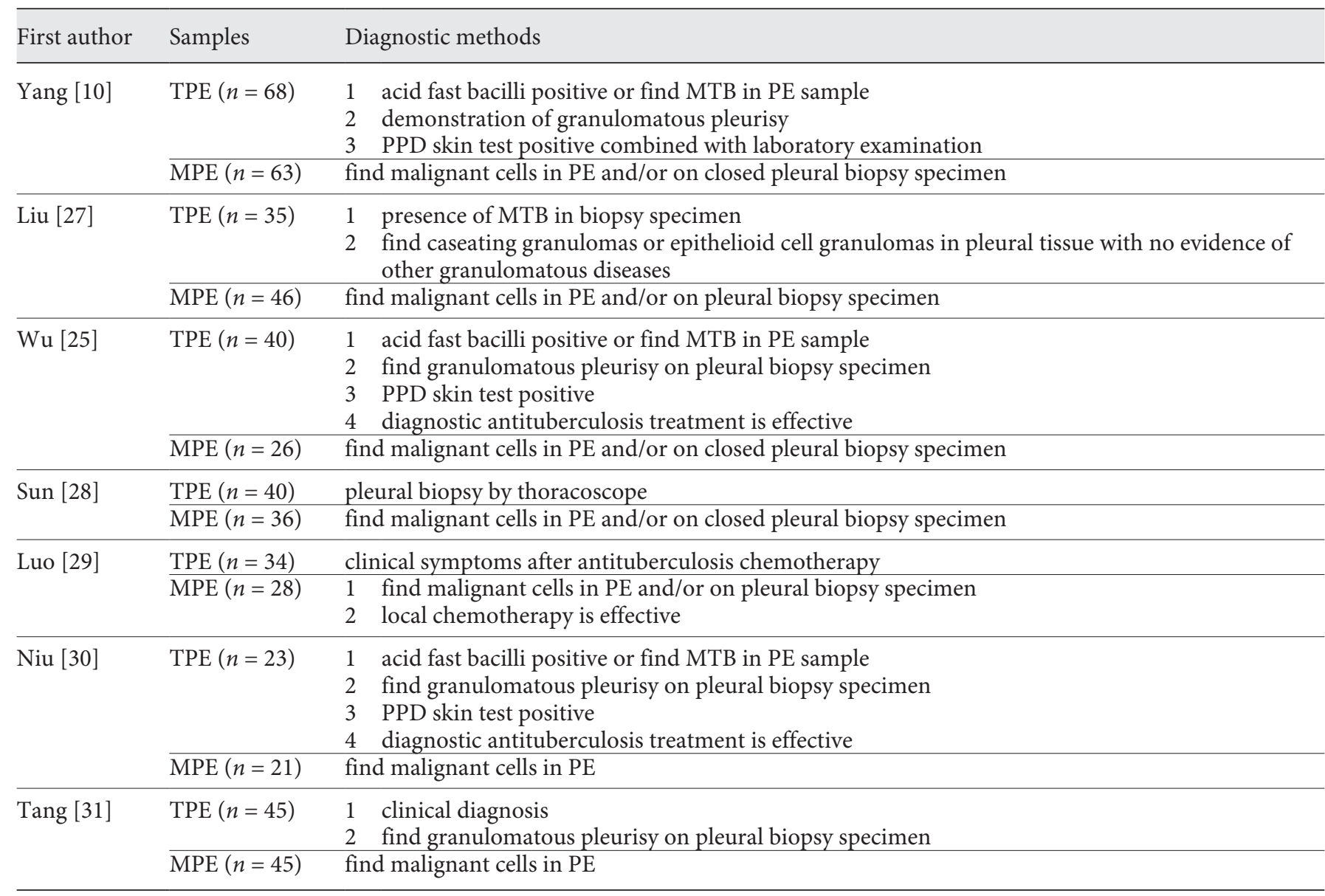

TPE, tuberculous pleural effusion; MPE, malignant pleural effusion; PE, pleural effusion. 
Fig. 2. Forest plot of the pooled sensitivity of IL-27 for the diagnosis of tuberculous pleural effusion from malignant pleural effusion.

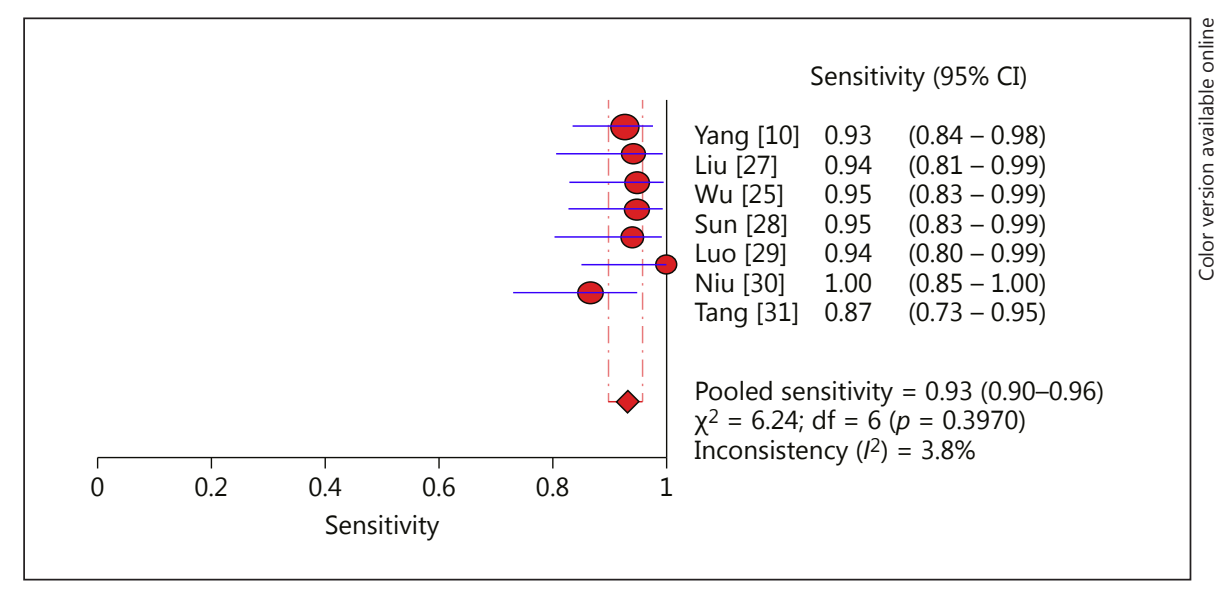

Fig. 3. Forest plot of the pooled specificity of IL-27 for the diagnosis of tuberculous pleural effusion from malignant pleural effusion.

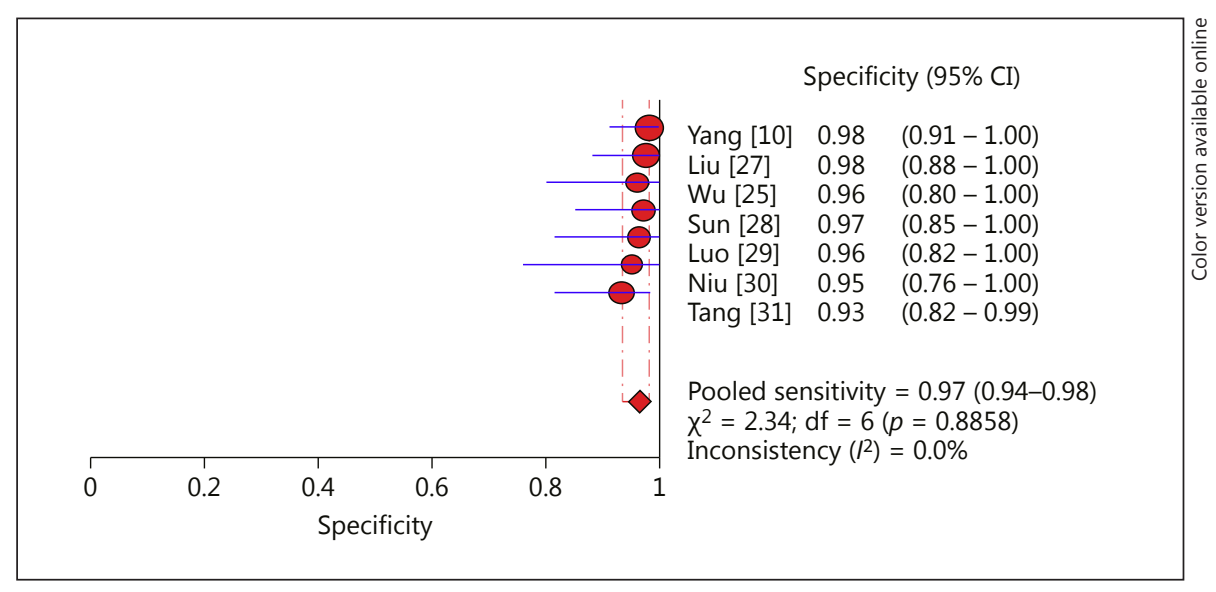

Data Extraction and Quality Assessment

Data extracted from the publications included authors, setting, publication year, test method, number of patients, cutoff values, data for two-by-two tables, SEN, SPE, and quality scores. We used two tools to assess the quality of publications: QUADAS [11] (quality assessment for studies of diagnostic accuracy) and STARD [12] (standards for reporting the accuracy of a diagnostic test).

\section{Data Analyses}

We performed the present meta-analysis for the accuracy of IL-27 by evaluating the overall SEN, SPE, positive likelihood ratio (PLR), negative likelihood ratio (NLR), diagnostic odds ratio (DOR), and plotting the summary receiver operating characteristic (SROC) curve. Post-test probability of TPE was obtained via: post-test probability $=$ post-test odds $/($ post-test odds +1$)$, where post-test odds $=($ prevalence $/[1-$ prevalence $]) \times$ likelihood ratio. We classified patients into two groups: TPE and MPE, and conducted the two-by-two table analysis. Inconsistency $\left(I^{2}\right)$ value was computed to indicate the statistically significant heterogeneity between the studies. If $I^{2}$ is less than $25 \%$, we consider the result nonsignificant for heterogeneity. In this case, the pooled results were conducted by the fixed-effect model. Conversely, all analyses were performed by the random-effect mode, and subgroup analyses should be conducted for the sake of exploring the potential causes of heterogeneity. Publication bias is a concern factor for meta- analysis of diagnostic studies. We examined the potential presence of this bias using the Deeks test [13]. A $p$ value $<0.05$ was considered to indicate a statistically significant result.

All analyses were operated by using the statistical software Meta-disc, version 1.4 (Meta-DiSc for Windows; XI Cochrane Colloquium; Barcelona, Spain) and Stata, version 12.0 (StataCorp, College Station, TX USA).

\section{Results}

\section{Study Characteristics and Quality Reports}

Following an independent review, we eventually pooled 7 publications, based on 285 patients with TPE and 265 patients with MPE were considered to be eligible for inclusion in the analysis. The characteristics and quality scores of these studies are outlined in Table 1. In all selected studies, TPE patients were diagnosed based on bacteriological, histological results or clinical course, including clinical feature, $\mathrm{PE}$ analysis, imaging presentation, and the responsiveness to antituberculosis chemotherapy. For the confirmation of MPE, 6 studies relied on 
Fig. 4. Forest plot of positive likelihood ratio of IL-27 for the diagnosis of tuberculous pleural effusion from malignant pleural effusion.

Fig. 5. Forest plot of negative likelihood ratio of IL-27 for the diagnosis of tuberculous pleural effusion from malignant pleural effusion.

Fig. 6. Forest plot of diagnostic odds ratio of IL-27 for the diagnosis of tuberculous pleural effusion from malignant pleural effusion.

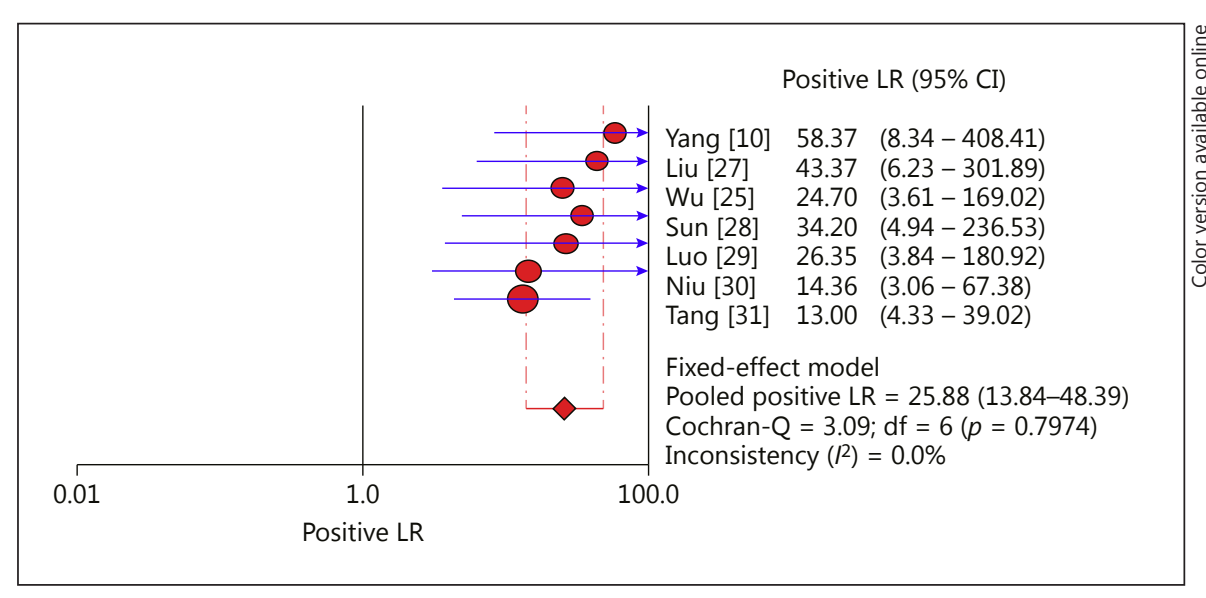

\section{Negative LR $(95 \%$ CI)}

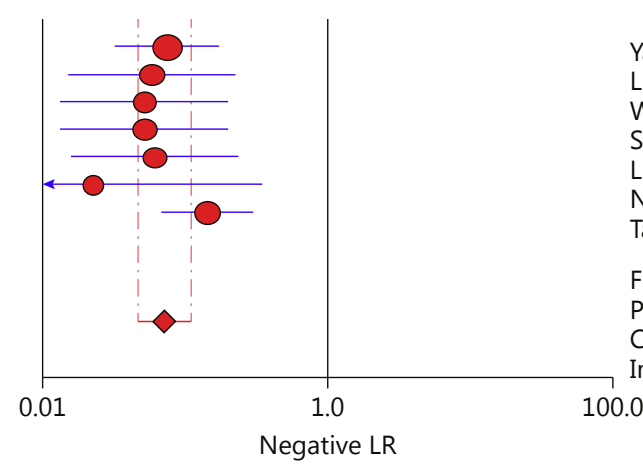

Yang [10] $0.07 \quad(0.03-0.17)$

Liu [27] $\quad 0.06 \quad(0.02-0.22)$

Wu [25] $\quad 0.05 \quad(0.01-0.20)$

Sun [28] $0.05 \quad(0.01-0.20)$

Luo [29] $0.06 \quad(0.02-0.23)$

Niu [30] $0.02 \quad(0.00-0.35)$

Tang [31] $\quad 0.14 \quad(0.07-0.30)$

Fixed-effect model

Pooled negative $L R=0.07(0.05-0.11)$

Cochran- $\mathrm{Q}=4.56 ; \mathrm{df}=6(p=0.6009)$

Inconsistency $\left(I^{2}\right)=0.0 \%$

Negative LR

Diagnostic OR (95\% CI)

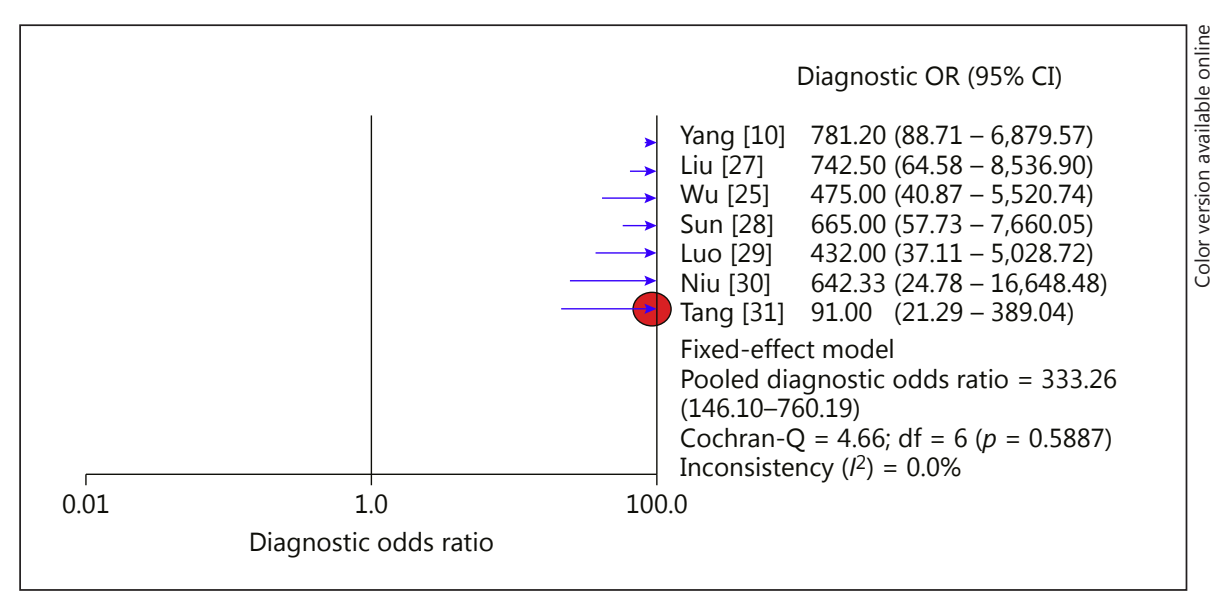

pathological diagnosis and 1 study on pathological diagnosis and clinical diagnosis (Table 2).

As shown in Table 1, the quality of research accuracy and reporting diagnostic accuracy of most studies were relatively good, as all studies had QUADAS scores $\geq 10$ and all STARD scores $\geq 12$.

Diagnosis and Prognosis of Pleural Effusion
SEN, SPE, PLR, NLR, DOR, and SROC Curve

We performed the analyses using the fixed-effect model. As shown in the forest plots (Fig. 2-6), the pooled SEN was 0.93 (95\% CI 0.90-0.96), while the pooled SPE was 0.97 (95\% CI 0.94-0.98). The PLR and NLR were 25.88 (95\% CI 13.84-48.39) and 0.07 (95\% CI 0.05-0.11), respectively. 
Table 3. Deeks test for publication bias

\begin{tabular}{lccccc}
\hline yb & Coefficient & SE & $t$ & $p>|t|$ & 95\% confidence interval \\
\hline Bias & -0.8342604 & 19.06563 & -0.04 & 0.967 & $-49.84401,48.17549$ \\
Intercept & 6.229881 & 2.157833 & 2.89 & 0.034 & $0.6829935,11.77677$ \\
\end{tabular}

yb, a general designation of a mathematical statistical model coefficient.

The DOR was 333.26 (95\% CI 146.10-760.19). Figure 7 shows that the SROC curve of IL-27 was placed very near the upper left corner, and the maximal joint SEN and SPE was 0.95 , while the area under the curve (AUC) was 0.99 .

\section{Post-Test Probability}

When pre-test prevalence of TPE is 10\% (an assumptive low-risk) and IL-27 result was positive, the estimated post-test probability was $75 \%$ (Fig. 8 ), whereas a negative test result nearly excluded TPE (post-test probability, $1 \%)$. For a patient with $50 \%$ pre-test risk, a positive test result of IL-27 could increase the probability to $96 \%$ (Fig. 9); on the contrary, the post-test probability was $6 \%$ with negative IL-27 test results.

\section{Heterogeneity and Publication Bias}

$I^{2}$ value of SEN, SPE, PLR, NLR, and DOR were less than $25 \%$, with all $p$ values $>0.05$, indicating an indistinctive heterogeneity between studies. In addition, the Deeks test for publication bias showed $p=0.51$ for IL-27, indicating that there was not a significant potential for publication bias (Table 3; Fig. 10).

\section{Discussion}

Making a differential diagnosis between TPE and MPE is a critical challenge. MPE, an advanced malignancy disease, with a median survival following diagnosis ranges from 3 to 12 months and is dependent on the stage and type of the underlying malignancy [14]. Histocytological analysis, for example discovering tumor cells in PE, remains necessary to confirm a malignant diagnosis and to define the histological tumor type [15]. At the moment, it should be emphasized that a definite diagnosis of TPE is achieved by a histological and pathogen test. Conventional methods, such as direct examination of PE by acid-fast staining, culture of $\mathrm{PE}$ and pleural biopsy, are not always helpful in making a definite diagnosis of TPE, since they

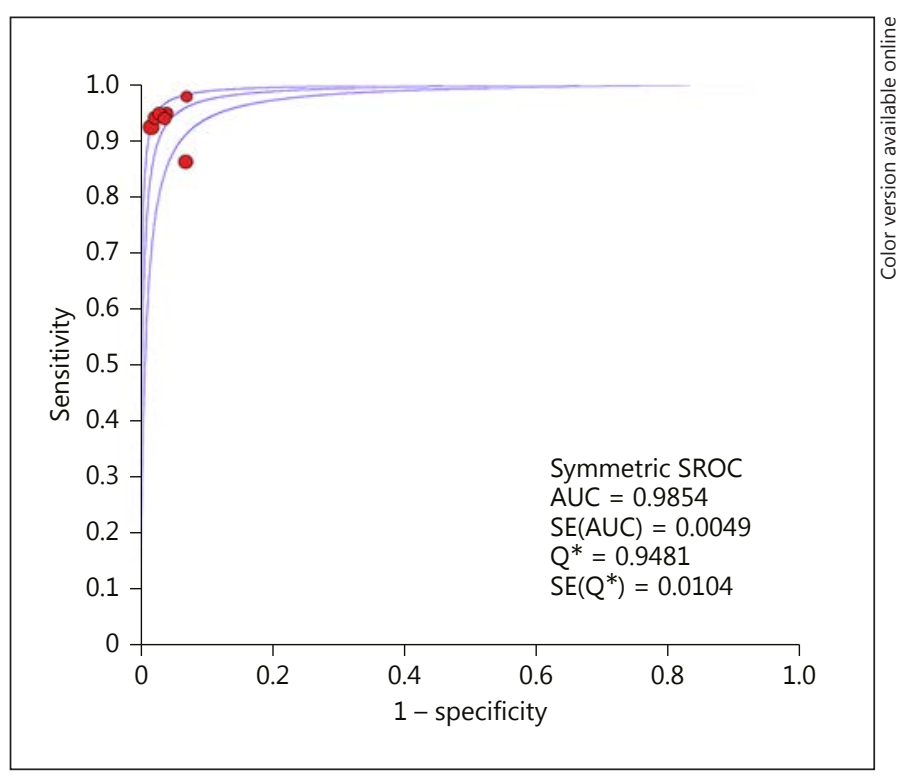

Fig. 7. Summary receiver operating characteristic curve for IL-27 about the diagnosis of tuberculous pleural effusion from malignant pleural effusion.

have limitations. Culturing M. tuberculosis is time-consuming (it usually take several weeks) while culturing PE has a low positive rate $(<5 \%)$ [16]. To confirm the diagnosis of TPE sometimes requires further invasive procedures such as medical thoracoscopy and thoracotomy. However, pleural biopsy is invasive, operator-dependent, and requires expertise [15] and pleurae of needle biopsy are not conclusive in $20-40 \%$ of patients with TPE [17, $18]$. These invasive procedures may cause various complications and may even increase morbidity and cannot be carried out in all hospitals. Although not perfect, these tests are widely considered the standard of diagnosis.

PE biomarkers have been proposed as assistants in the diagnosisofTPE, including those of ADA andinterferon- $\gamma$, the levels of which are all significantly higher in TPE than in MPE. In one of the published meta-analyses that summarizes the overall accuracy of ADA in the diagnosis of
Liu/Yu/Wang/Wang/Wang 


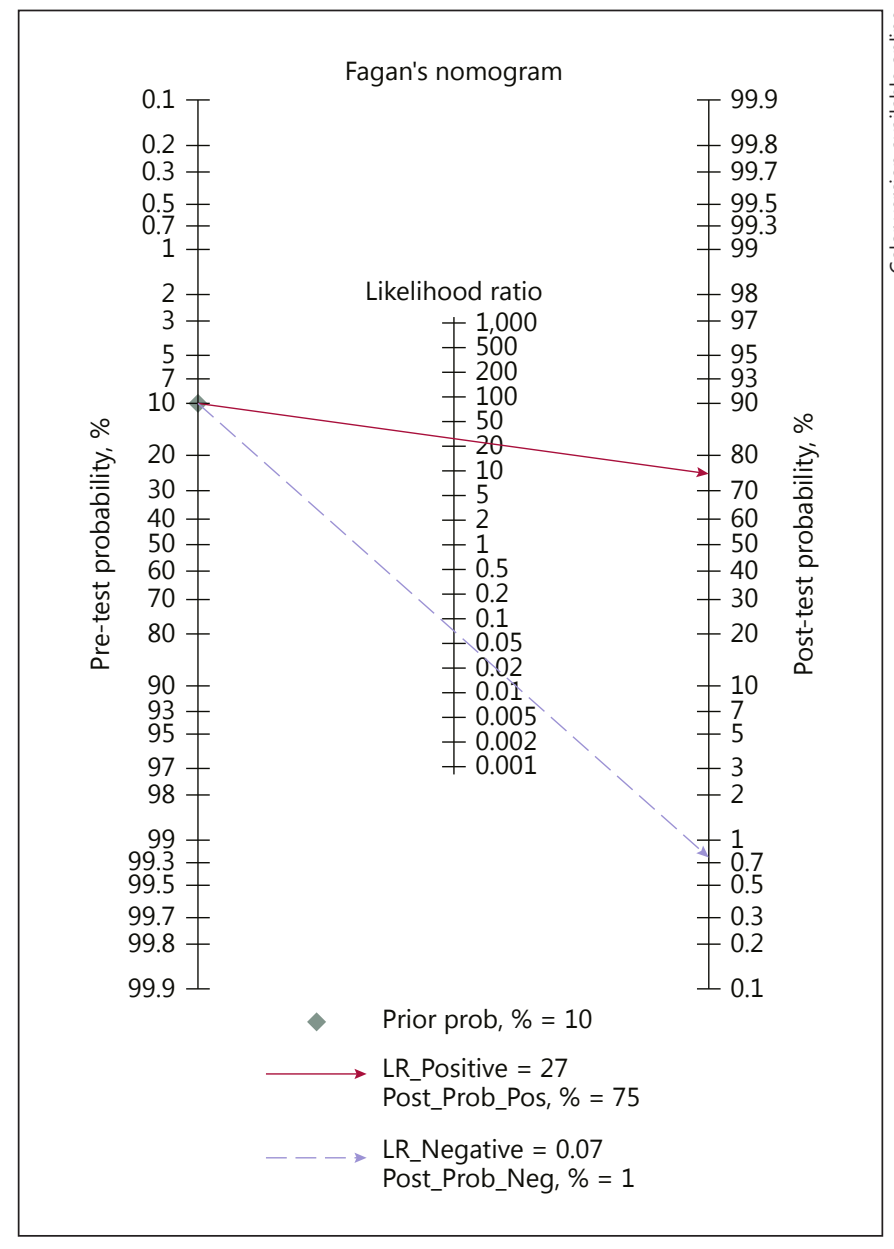

Fig. 8. Fagan's nomogram for post-test probability of tuberculous pleural effusion at $10 \%$ pre-test probability.

TPE compared to non-TPE, SEN was 0.92 (95\% CI 0.90 0.93), SPE was 0.90 (95\% CI 0.89-0.91), PLR was 9.03 (95\% CI 7.19-11.35), NLR was 0.10 (95\% CI 0.07-0.14), and DOR was 110.08 (95\% CI 69.96-173.20) [19]. In another published meta-analysis about interferon- $\gamma$ for the diagnosis of TPE from non-TPE, the pooled SEN, SPE, PLR, NLR, and DOR were 0.89 (95\% CI 0.87-0.91), 0.97 (95\% CI 0.96-0.98), 23.45 (95\% CI 17.31-31.78), 0.11 (95\% CI 0.07-0.16), and 272.7 (95\% CI 147.5-504.2), respectively [20]. The present meta-analysis demonstrates that the pooled SEN of IL-27 assay was 0.93 (95\% CI 0.900.96), while pooled SPE was 0.97 (95\% CI 0.94-0.98). It can be observed that the diagnostic performance of IL-27 for TPE is superior to ADA and interferon- $\gamma$.

The SROC curve and the AUC indicate the overall summary of test efficiency, and present the tradeoff between SEN and SPE. Our data show that the maximal

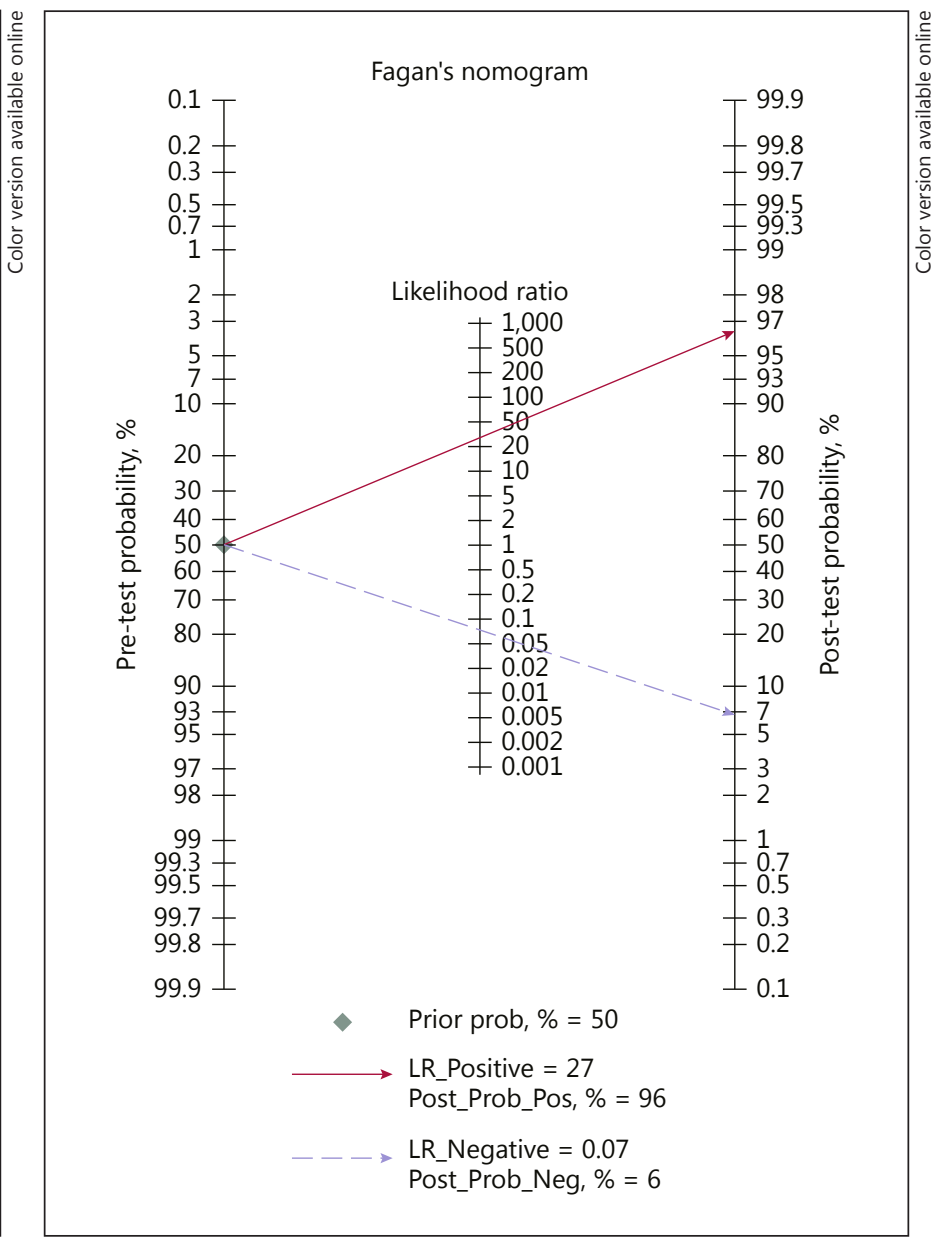

Fig. 9. Fagan's nomogram for post-test probability of tuberculous pleural effusion at $50 \%$ pre-test probability.

joint SEN and SPE was 0.95, while the AUC was 0.99, indicating a very high overall accuracy of TPE diagnosis.

The DOR is a single indicator of test accuracy that combines the data from SEN and SPE into a single number [21]. The DOR of a test is the ratio of the odds of positive test results in the diseased relevant to the odds of positive test results in the nondiseased. The value of a DOR fluctuates from 0 to infinity, with higher values implying preferable distinguishing test performance. A DOR of 1.0 manifests that the test does not differentiate between patients with the sickness and those without it. In our meta-analysis, we have found that the DOR was 333.26 (95\% CI 146.10-760.19), also indicating a high and satisfactory overall accuracy of IL-27 in the discriminate diagnosis between TPE and MPE.

As measures, likelihood ratios (LR) take into account the interaction between SEN and SPE in their calculation. 


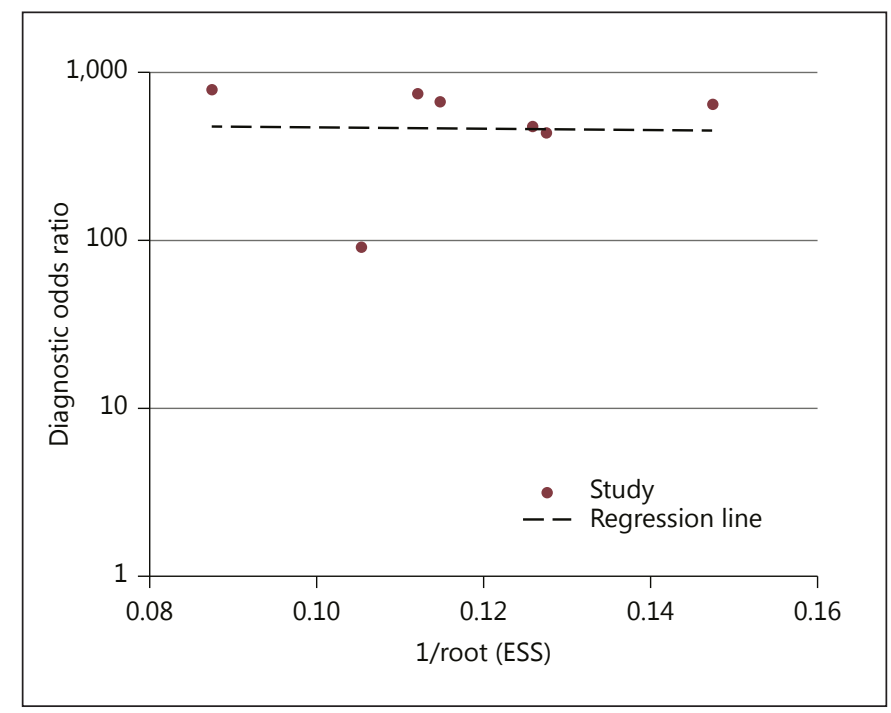

Fig. 10. Deeks graph for the assessment of potential publication bias in IL-27. $\log$ odds ratio versus $1 /$ sqrt(effective sample size) (Deeks test).

In most cases, PLR $>10$ and NLR $<0.1$ are considered convincing evidence to rule in or rule out diagnoses, respectively [22]. The PLR value of 25.88 (95\% CI 13.84-48.39) in the present meta-analysis indicates that patients with TPE have an about 26-fold higher chance of being IL-27 test-positive compared with patients with MPE. These high odds would be considered high enough to begin or to continue antituberculosis treatment of the patients who were highly suspected to have TPE in the clinical practice, especially in the case of the absence of any evidence of malignancies. Of our data, NLR was found to be 0.07 (95\% CI 0.05-0.11). This means that, if the IL-27 test result was negative, the probability that the patient has TPE is 7\%, which is low enough to rule out TPE. Although the IL-27 test can rule out TPE, for the need to continue treatment, the detection of IL-27 is not a substitute for biopsy, as pathological classification and genetic testing of some patients with pleural metastasis may be required for the diagnosis and treatment as early as possible, such as targeted drug treatment. It reminds us of the necessity to apply a biopsy of pleura, lung tissue or lymph node to hunt for proof of malignancies. After all, except TB, the most likely result is malignancy if a patient with exudative PE has predominantly lymphocytes. Sun et al. [23] concluded that endobronchial ultrasound elastography is a promising method that is used for the identification of benign and malignant lymph nodes with high SEN, SPE, and predictive accuracy, which may offer important supplementary information in endobronchial ultrasound- guided transbronchial needle aspiration cases. Karpathiou et al. [24] also raise the issue that pleural frozen sections are a valuable and accurate tool in immediately diagnosing malignancy, permitting further operations like pleurodesis in the meantime.

In regard to pre-test probability, it is a vital factor to interpret IL-27 results when making a clinical decision. Our meta-analysis exhibited that when the predicted probability of TPE is low (10\%), the overall post-test probability of being IL-27 test-positive is inadequate to serve as a "rule in" diagnostic method. However, the probability could be raised to $96 \%$ when there were positive test results of IL-27 for TPE in high-prevalence settings (50\%), meaning that further diagnostic measures are inevitable for the diagnosis of TPE, especially the invasive tests. In low- or high-prevalence settings, if the result of IL-27 is negative, the post-test probabilities are low enough (always $<6 \%$ ) to rule out TPE.

The meta-analysis demonstrates that IL-27 has a very high accuracy in the diagnosis between TPE and MPE, which is obviously superior to ADA and interferon- $\gamma$. The combination of IL-27 with other markers in PE may aid the establishment of the definite diagnosis of TPE. For instance, the combination of IL-27, ADA and interferon- $\gamma$ has been reported to have a high diagnostic performance, with both SEN and SPE of $100 \%$ for TPE [25]. However, Skouras et al. [26] put forward that the accuracy of IL-27 and ADA in the diagnosis of TPE is relative to the prevalence of tuberculosis. The viewpoint of his research is that only at the low tuberculosis prevalence does IL-27 or ADA present a low false-negative ratio $(<5 \%)$, which is does no correspond to our conclusion. We still need numerous studies to evaluate the diagnostic value of IL-27 for the TPE diagnosis in areas with different tuberculosis prevalence and carry out further research of IL-27*ADA (the product of IL-27 and ADA), which shows a low false-negative ratio $(<1 \%)$ at any tuberculosis morbidity area [26].

To our knowledge, this paper is the first meta-analysis that summarizes the discrimination diagnostic value of IL-27 between TPE and MPE. There are still some limitations to our study. First, even though we tried our best to retrieve any related studies and obtain additional data from investigators, it seems inevitable that some missing and unpublished data may still exist. Furthermore, languages of included studies are limited to English and Chinese, which may result in a language bias. Third, included studies adopt different diagnostic criteria while diagnosing TPE, which could cause misclassification bias. Lastly, blind methods were not mentioned in any enrolled studies, which may have led to a bias result. 


\section{Conclusion}

The current data suggest that even though it is not a gold standard, the detection of PE IL-27 has very high accuracy for differentiating TPE from MPE. The results of the IL-27 test should be interpreted in parallel with clinical findings and the results of conventional tests including histological and microbiological examination.

\section{Financial Disclosure and Conflicts of Interest}

None of the authors have a financial relationship with a commercial entity that has an interest in the subject of this paper.

\section{Funding Sources}

This work was supported in part by grants from the National Natural Science Foundation of China (No. 81600070).

\section{References}

1 Porcel JM, Light RW: Pleural effusions. Dis Mon 2013;59:29-57.

2 Light RW: Clinical practice. Pleural effusion. N Engl J Med 2002;346:1971-1977.

-3 Trajman A, Pai M, Dheda K, et al: Novel tests for diagnosing tuberculous pleural effusion: what works and what does not? Eur Respir J 2008;31:1098-1106.

4 Yang Y, Liu YL, Shi HZ: Diagnostic accuracy of combinations of tumor markers for malignant pleural effusion: an updated meta-analysis. Respiration 2017;94:62-69.

5 Pflanz S, Timans JC, Cheung J, et al: IL-27, a heterodimeric cytokine composed of EBI3 and $\mathrm{p} 28$ protein, induces proliferation of naive CD4+ T cells. Immunity 2002;16:779790.

6 Batten M, Ghilardi N: The biology and therapeutic potential of interleukin 27. J Mol Med (Berl) 2007;85:661-672.

7 Hisada M, Kamiya S, Fujita K, et al: Potent antitumor activity of interleukin-27. Cancer Res 2004;64:1152-1156.

8 O'Dwyer MJ, Mankan AK, White M, et al: The human response to infection is associated with distinct patterns of interleukin 23 and interleukin 27 expression. Intensive Care Med 2008;34:683-691.

9 Robinson CM, Nau GJ: Interleukin-12 and interleukin-27 regulate macrophage control of Mycobacterium tuberculosis. J Infect Dis 2008;198:359-366.

10 Yang WB, Liang QL, Ye ZJ, et al: Cell origins and diagnostic accuracy of interleukin 27 in pleural effusions. PLoS One 2012;7:e40450.

11 Whiting P, Rutjes AW, Reitsma JB, et al: The development of QUADAS: a tool for the quality assessment of studies of diagnostic accuracy included in systematic reviews. BMC Med Res Methodol 2003;3:25.

12 Bossuyt PM, Reitsma JB, Bruns DE, et al: Towards complete and accurate reporting of studies of diagnostic accuracy: the STARD initiative. BMJ 2003;326:41-44.

13 Deeks JJ, Macaskill P, Irwig L: The performance of tests of publication bias and other sample size effects in systematic reviews of diagnostic test accuracy was assessed. J Clin Epidemiol 2005; 58:882-893.

14 Roberts ME, Neville E, Berrisford RG, et al: Management of a malignant pleural effusion: British Thoracic Society Pleural Disease Guideline 2010. Thorax 2010;65(suppl 2):i32-i40.

15 Porcel JM, Azzopardi M, Koegelenberg CF, et al: The diagnosis of pleural effusions. Expert Rev Respir Med 2015;9:801-815.

16 Zhou Q, Chen YQ, Qin SM, et al: Diagnostic accuracy of T-cell interferon-gamma release assays in tuberculous pleurisy: a meta-analysis. Respirology 2011;16:473-480.

-17 Escudero BC, Garcia CM, Cuesta CB, et al: Cytologic and bacteriologic analysis of fluid and pleural biopsy specimens with Cope's needle. Study of 414 patients. Arch Intern Med 1990;150:1190-1194.

18 Epstein DM, Kline LR, Albelda SM, et al: Tuberculous pleural effusions. Chest 1987;91: 106-109.

19 Liang QL, Shi HZ, Wang K, et al: Diagnostic accuracy of adenosine deaminase in tuberculous pleurisy: a meta-analysis. Respir Med 2008;102:744-754.

20 Jiang J, Shi HZ, Liang QL, et al: Diagnostic value of interferon-gamma in tuberculous pleurisy: a metaanalysis. Chest 2007;131: 1133-1141.

21 Glas AS, Lijmer JG, Prins MH, et al: The diagnostic odds ratio: a single indicator of test performance. J Clin Epidemiol 2003;56:11291135.

22 Jaeschke R, Guyatt GH, Sackett DL: Users' guides to the medical literature. III. How to use an article about a diagnostic test. B. What are the results and will they help me in caring for my patients? The Evidence-Based Medicine Working Group. JAMA 1994;271:703-707.

23 Sun J, et al: Endobronchial ultrasound elastography for evaluation of intrathoracic lymph nodes: a pilot study. Respiration 2017; 93:327-338.

24 Karpathiou G, et al: Frozen sections in pleural pathology: a valuable tool. Respiration 2017; 94:45-51.

25 Wu YB, Ye ZJ, Qin SM, et al: Combined detections of interleukin 27, interferon-gamma, and adenosine deaminase in pleural effusion for diagnosis of tuberculous pleurisy. ChinMed J (Engl) 2013;126:3215-3221.

26 Skouras VS, Magkouta SF, Psallidas I, et al: Interleukin-27 improves the ability of adenosine deaminase to rule out tuberculous pleural effusion regardless of pleural tuberculosis prevalence. Infect Dis (Lond) 2015;47: 477-483.

27 Liu YL, Wu YB, Zhai K, et al: Determination of interleukin 27-producing CD4(+) and CD8(+) T cells for the differentiation between tuberculous and malignant pleural effusions. Sci Rep 2016;6:19424.

28 Sun M, Yan D, Jiang S, et al: Diagnostic value of interleukin-27 in tuberculous pleural effusion. Zhonghua Yi Xue Za Zhi 2014;94:26412644.

29 Luo JH, Wu N: Value of the combination of IL-27, ADA and INR- $\gamma$ for the differential diagnosis of tuberculous and malignant pleural effusion. Hebei Med J 2015;37:512-514.

30 Niu CM: Interleukin-27 in Tuberculous and Malignant Pleural Effusion. Huazhong University of Science and Technology, 2012.

31 Tang J, Zhang XM, Wan F, et al: Value of the combination of three cytokines for the differential diagnosis of tuberculous and malignant pleural effusion. Chin J Antituberc 2017;39: 76-81. 